\title{
Vasculite ANCA associata in un paziente con trombofilia ereditaria
}

\author{
Giovanni Salvati, Mario Paracuollo, Roberta Rossano, Marco Terribile
}

U.O.C. di Nefrologia e Dialisi, Ospedale dei Pellegrini, Napoli

\begin{abstract}
ANCA ASSOCIATED VASCULITIS IN PATIENT WITH HEREDITARY THROMBOPHILIA
Abstract. We here describe the clinical case of a patient who was diagnosed with worsening renal failure due to suspected vasculitis. Our chemical and histological findings confirmed the vasculitis with an uncertain clinical diagnosis either of Wegener granulomatosis or microscopic polyangiitis. Either way, this uncertainty did not influence the therapy, which, according to the guidelines, is the same for the two diseases. The patient was treated with methylprednisolone, cyclophosphamide, and plasmapheresis. However, the clinical outcome on renal function was not satisfactory, and start of hemodialysis was necessary. In this phase, repeated thrombotic episodes occurred, despite they never did before. Consequently, we performed blood tests on coagulation and thrombosis that revealed a condition of hyperhomocysteinemia. We then performed genetic testing and showed "hereditary thrombophilia" related to 3 genetic alterations. Treatment with heparin and folic acid has prevented the recurrence of thrombotic events. In conclusion, in our patient the picture of "ANCA-associated vasculitis" was developed on a "habitus of hereditary thrombophilia." Therefore, the treatment of vasculitis alone was found unsatisfactory. The combination with the other disease has probably had a negative impact on renal prognosis, and its presence represented a confounding element for the clinical picture.
\end{abstract}

Key words: Anti-PR3 ANCA, Vasculitis, Hyperhomocysteinemia, Hereditary thrombophilia

Conflict of interest: None.

Financial support: None.

Accettato: 9 Luglio 2014

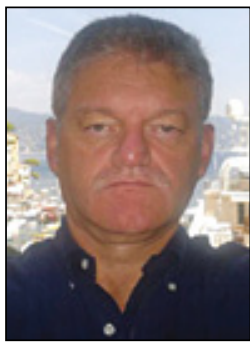

Giovanni Salvati

\section{Caso Clinico}

Paziente di anni 62, muratore, in buona salute fino ad Agosto 2012, epoca in cui per circa 3 mesi presentava febbricola, mialgia e poliartralgia. Era trattato prima con FANS e poi con steroidi.

A Dicembre comparivano papule e petecchie cutanee accompagnate da tosse ed emottisi. Presentava anche insufficienza renale con creatininemia di $2.4 \mathrm{mg} / \mathrm{dL} \%$.

Si ricoverava pertanto presso la Medicina di un Ospedale cittadino.

Effettuava radiografia del torace che evidenziava "Addensamenti multipli con margini mal definiti e marcate aree di opacità parenchimali a chiazze e accentuazione della trama vascolare tipo reticolo micronodulare specialmente a sede ilo-basale destra".

La funzionalità renale risultava ulteriormente compromessa con azotemia di $130 \mathrm{mg} / \mathrm{dL}$, creatininemia di $5.6 \mathrm{mg} / \mathrm{dL}$ e microematuria e proteinuria in range non nefrosico. Erano presenti anemia con $\mathrm{Hb}$ di $10 \mathrm{~g} / \mathrm{dL}$ e indici infiammatori alterati con VES $121 \mathrm{~mm}$, PCR $47 \mathrm{mg} / \mathrm{dL}, \mathrm{C}_{3} 200 \mathrm{mg} / \mathrm{dL} \mathrm{e} \mathrm{C}_{4} 45 \mathrm{mg} / \mathrm{dL}$.
Concomitava ipertensione arteriosa. Era diagnosticato "Processo broncopneumonico acuto bilaterale con intensa interstiziopatia, IRA, anemia e ipertensione arteriosa".

Il paziente era trattato con antibiotici e antipertensivi ed era poi dimesso dopo alcune settimane.

Non essendosi risolta l'insufficienza renale si ricoverava il 24/01/13 presso la nostra U.O.C. con la diagnosi di "IRA da sospetta vasculite".

Gli esami effettuati all'atto del ricovero sono riportati nella Tabella I.

Erano presenti anemia con $\mathrm{Hb}$ di $7.7 \mathrm{~g} / \mathrm{dL}$, leucocitosi, trombocitosi, ematuria microscopica, proteinuria e insufficienza renale. La ricerca dei marcatori immunologici dava positività per gli ANCA anti-PR3.

Per l'acuzie del quadro clinico si sottoponeva il paziente a Biopsia Renale.

Il referto istologico:

Nel frustolo esaminato si osservano 13 glomeruli, uno dei quali in scleroialinosi subtotale.

Gli altri glomeruli presentano in vario grado quadri che vanno dalla necrosi segmentaria a quella più diffusa. Necrosi segmentaria del flocculo si osserva in 7 glofmeruli, con proliferazioni epiteliali a semiluna e adesione flocculo-capsulare 
TABELLA I - GLI ESAMI EMATOCHIMICI DEL PAZIENTE

\begin{tabular}{|c|c|c|c|}
\hline & $\begin{array}{c}\text { AL RICOVERO } \\
26 / 01 / 13 \\
\end{array}$ & $\begin{array}{c}\text { INIZIO DIALISI } \\
15 / 02 / 13 \\
\end{array}$ & $\begin{array}{c}\text { TROMBOFLEBITE } \\
15 / 3 / 13 \\
\end{array}$ \\
\hline CREATININEMIA $m g / d L$ & 5.9 & 9.16 & 9.76 \\
\hline PROTIDEMIA $g / d L$ & 7.6 & 6.5 & 7.3 \\
\hline ALBUMINA $\%$ & 41.5 & 43 & 50.2 \\
\hline Alfa $2 \%$ & 16.5 & 15.8 & 15 \\
\hline Gamma \% & 19.4 & 20.9 & 14.2 \\
\hline FERRITINA $n g / m l$ & 2701 & 1080 & 870 \\
\hline $\mathrm{HB} g / d L$ & 7.7 & 10.2 & 10.5 \\
\hline G. BIANCHI $\mathrm{mm}^{3}$ & 21100 & 28000 & 11800 \\
\hline PIASTRINE $\mathrm{mm}^{3}$ & 505000 & 479000 & 173000 \\
\hline Р.Т. $\%$ & 77.4 & 87 & 91.4 \\
\hline PTT “ & 32.2 & 33 & 36.2 \\
\hline FIBRINOGENO $m g / d L$ & 501 & 480 & 153 \\
\hline D-DIMERO $\mu g / L$ & 450 & & 7742 \\
\hline PROTEINURIA $24 \mathrm{H} m g$ & 870 & 340 & 356 \\
\hline MICROEMATURIA & presente & presente & presente \\
\hline VES & 96 & 87 & 78 \\
\hline $\mathrm{PCR} m g / d L$ & 7.5 & 5 & 3 \\
\hline REUMA TEST $U I / m L$ & 49 & 38 & 24 \\
\hline CRIOGLOBULINE & assenti & assenti & assenti \\
\hline $\operatorname{Ig} \mathrm{G} m g / d L$ & 1046 & 1300 & 1430 \\
\hline $\operatorname{Ig} \mathrm{A} m g / d L$ & 159 & 198 & 199 \\
\hline $\operatorname{Ig} \mathrm{M} m g / d L$ & 88 & 123 & 133 \\
\hline C $3 \mathrm{mg} / \mathrm{dL}$ & 198 & 170 & 143 \\
\hline C $4 m g / d L$ & 40 & 37 & 32 \\
\hline ANA & assenti & assenti & assenti \\
\hline anti DNA & assenti & assenti & assenti \\
\hline PR3 ANCA $U I / m L$ & 113 & 100 & 35 \\
\hline MPO ANCA $U I / m L$ & 3 & 5 & 3 \\
\hline PROTEINA C \% & & & 144 \\
\hline PROTEINA S \% & & & 128 \\
\hline OMOCISTEINA $\mu \mathrm{mol} / \mathrm{L}$ & & & 156 \\
\hline
\end{tabular}

di vari grado ed estensione; sono presenti intenso infiltrato infiammatorio con accumulo di leucociti, che zaffano e comprimono il flocculo e arteriole con lume ristretto per endotelio rigonfio; nelle anse sane dei glomeruli non si osservano espansione o proliferazione del mesangio o alterazioni della MB glomerulare. Sono presenti nei frustoli esaminati 2 arteriole con lume ristretto per endotelio rigonfio. In una di esse il lume risulta obliterato.

L'interstizio presenta denso infiltrato infiammatorio leucocitario peri-glomerulare.

Un glomerulo mostra rottura della capsula del Bowmann, con marcata flogosi circostante.
I tubuli presentano diffusi aspetti di necrosi e atrofia o di rigenerazione e sono anch'essi infiltrati spesso da elementi della flogosi.

L'immunofluorescenza è completamente negativa, escluso un piccolo deposito di fibrina osservato in un glomerulo e nella sua area peri-glomerulare.

Le lesioni descritte si presentavano in fase attiva con aspetti perlopiù focali e definivano un quadro di "Glomerulonefrite necrotizzante extracapillare pauciimmune" tipico delle vasculiti ANCA associate.

Alla luce dei reperti laboratoristici e del quadro clinico segnalato, si orientava la diagnosi verso la Granulomato- 
TABELLA II - TERAPIA DELLA VASCULITE ANCA ASSOCIATA ESEGUITA NEL NOSTRO PAZIENTE (1-3)

\begin{tabular}{|c|c|}
\hline & Induzione \\
\hline Cortisone & $\begin{array}{l}\text { Metilprednisolone: boli e.v. di } 1000 \mathrm{mg} / \text { die }(12 \mathrm{mg} / \mathrm{kg} \\
\text { p.c.) x } 3 \mathrm{gg} \text {. seguiti da } \\
\text { Prednisone } 1 \mathrm{mg} / \mathrm{kg} \text { p.c./die per os la } 1^{\mathrm{a}} \text { sett., } \\
\text { poi } 0.75 \mathrm{mg} / \mathrm{kg} \text { p.c. } / \text { die } \text { per os la } 2^{\mathrm{a}} \text { sett., } \\
\text { poi } 0.5 \mathrm{mg} / \mathrm{kg} \text { p.c. } / \text { die } \text { per os per } 2 \text { sett., } \\
\text { poi } 0.4 \mathrm{mg} / \mathrm{kg} \text { p.c. } / \text { die } \text { per os per } 2 \text { sett., } \\
\text { poi } 0.3 \mathrm{mg} / \mathrm{kg} \text { p.c. } / \text { die } \text { per os per } 2 \text { sett. }\end{array}$ \\
\hline Ciclofosfamide & $\begin{array}{l}\text { Boli e.v. di } 500 \mathrm{mg}(7.5 \mathrm{mg} / \mathrm{kg} \text { p.c.) ogni } 15 \mathrm{gg} . \mathrm{X} 3 \\
\text { boli, poi } 1 \text { bolo ogni } 21 \mathrm{gg} \text {. }\end{array}$ \\
\hline Plasmaferesi & $\begin{array}{l}\text { Associata dopo due settimane, con } 6 \text { sedute in } 2 \text { sett. e } \\
\text { scambi di } 40-60 \mathrm{~mL} / \mathrm{kg} / \mathrm{seduta}\end{array}$ \\
\hline & Mantenimento \\
\hline
\end{tabular}

Prednisone $0.25 \mathrm{mg} / \mathrm{kg}$ p.c./die per os

Ciclofosfamide $1 \mathrm{mg} / \mathrm{kg}$ p.c./die per os per un mese sostituita poi con azatioprina $1 \mathrm{mg} / \mathrm{kg}$ p.c./die per os

si di Wegener o la Poliangioite microscopica, essendo le lesioni morfologiche renali del tutto simili in entrambe le patologie.

Si iniziava una terapia intensiva cortico-steroidea/ciclofosfamide, alla quale si associava poi la plasmaferesi, vista l'evoluzione negativa del quadro clinico.

La terapia immunosoppressiva effettuata era quella definita dalle Linee Guida per le vasculiti ANCA associate (Tab. II). Alla terapia con ciclofosfamide si associava Mesna che lega l'acroleina, metabolita tossico della ciclofosfamide, e ne riduce la tossicità vescicale.

Grazie a questa terapia, continuata poi con dosaggi più bassi per la fase di mantenimento successiva, il paziente risultava in remissione a livello respiratorio e cutaneo, ma non migliorava la funzionalità renale.

Il paziente doveva iniziare a Febbraio la terapia dialitica. I parametri ematochimici di questa fase della malattia sono evidenziati nella Tabella I.

Aveva proseguito per altri due mesi la terapia immunosoppressiva e la plasmaferesi ma aveva dovuto continuare l'emodialisi, non essendo migliorata la funzionalità renale.

Compariva però una patologia trombotica, mai precedentemente manifestatasi, che dominava il quadro successivo.

Il paziente, in attesa del confezionamento di FAV, aveva dializzato con cateteri venosi femorali ripetutamente sostituiti e cambiati di posizione per malfunzionamento.

Erano comparse prima una trombosi dell'arto inferiore destro e successivamente, dopo rimozione del catetere e nuovo posizionamento nella vena femorale sinistra, una trombosi venosa profonda all'arto inferiore sinistro.

Erano stati effettuati esami ematochimici sia generali che specifici per i problemi della coagulazione, anch'essi riportati nella Tabella I.

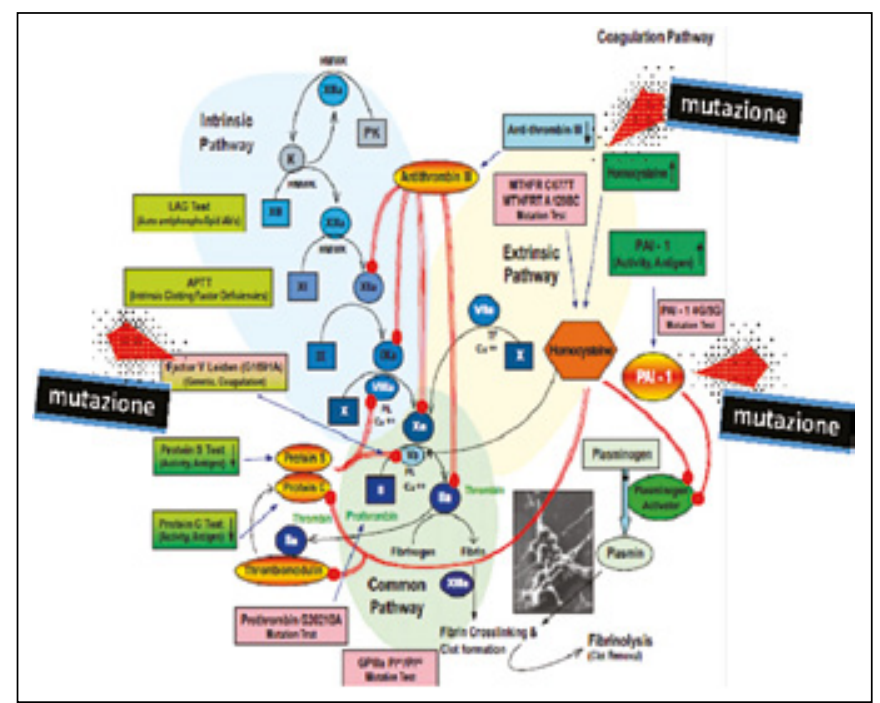

Fig. 1 - Le 3 alterazioni genetiche responsabili dello stato trombofiliaco.

Si erano rilevati altissimi livelli di omocisteina. Pertanto si era sospettato uno stato trombofiliaco ereditario.

Era stato effettuato uno studio genetico che aveva portato alla diagnosi di "Trombofilia familiare" legata a 3 alterazioni genetiche. Era presente infatti omozigosi mutata di PAI-1, omozigosi mutata di MTHFR A1298C ed eterozigosi mutata del Fattore V di Leiden (Fig. 1). Il paziente era stato sottoposto a terapia cronica con eparina sottocute e ad alte dosi di acido folico. I fenomeni trombotici non si sono più manifestati successivamente.

Lo studio genetico è stato successivamente esteso alla famiglia tra cui due figlie che avevano avuto nel passato aborti spontanei. Esse sono risultate portatrici di 2 delle 3 alterazioni genetiche presenti nel padre. Grazie a questa evidenza una delle due figlie, che era in quel periodo incinta, sottoposta a terapia eparinica a più alte dosi di acido folico, è riuscita a portare a termine la gravidanza spontaneamente.

\section{Discussione}

Il paziente aveva contemporaneamente sia una vasculite che uno stato trombofiliaco su base genetica con tre mutazioni diverse. Nella Tabella III sono evidenziati i possibili stati trombofiliaci ereditari.

Nel nostro paziente erano presenti 3 diverse mutazioni.

- Eterozigosi per il Fattore V Leiden mutazione G1691A La frequenza di eterozigosi per il fattore $\mathrm{V}$ di Leiden in Italia è pari al 2-3\% e in Europa al 6-8\% della popolazione (4). Questa alta frequenza si spiega con il fatto che tale mutazione, nel corso della storia dell'evoluzione, ha rappresentato un vantaggio, consentendo un ridotto rischio di emorragie in caso di ferite. Comunque i soggetti eterozigoti presentano un incremento del fattore di rischio trombotico, perlopiù di tipo venoso, di 4-8 volte maggiore rispetto alla popolazione generale $(5,6)$. Infatti la mutazione del fattore $\mathrm{V}$ rende insensibile e resistente il fattore $\mathrm{V}$ verso il proprio antagonista, la proteina 


\section{TABELLA III - STATI TROMBOFILIACI EREDITARI}

- Antitrombina: deficienza (antitrombina III no)

- Anormalità nei sistemi di proteina $\mathrm{C}$ e proteina $\mathrm{S}$

- proteina $\mathrm{C}$ deficienza

- proteina S deficienza

- anormalità nella trombomodulina

- Resistenza all'attivazione della proteina C (FV G1691A)

- Ipertrombinemia (fattore II G20210A)

- Disfibrinogenemia (beta-fibrinogeno G455A)

- Anormalità del sistema fibrinolitico

- ipo- o dis- plasminogenemia

- elevati livelli dell'inibitore del plasminogeno (PAI $14 \mathrm{G} / 5 \mathrm{G}$ )

- decreased tissue plasminogen activator

- Iperomocisteinemia (MTHFR, CBS)

- Eparina cofattore II, deficienza

- Elevati livelli di glicoproteine ricche in istidina

- Fattore XII, deficienza

C attivata APC (7).

\section{- Polimorfismo A1298C della MTHFR}

Ha una frequenza in omozigosi dell' $11 \%$ ed è responsabile di una ridotta attività enzimatica.

Il MTHFR (metilene-tetraidrofolato reduttasi) ha un ruolo essenziale nel metabolismo dell'omocisteina; catalizza la trasformazione dell'omocisteina in metionina mediante rimetilazione. Per la mutazione MTHFR A1298C ne consegue un aumento dei livelli circolanti di omocisteina (8), con conseguente danno endoteliale e aumentato rischio di malattia cardiovascolare.

\section{- Polimorfismo del PAI-1}

È del tipo "inserzione-delazione" di una G (4G/5G). L'allele $4 \mathrm{G}$, se presente in omozigosi, come nel caso del nostro paziente, è associato a livelli più alti di PAI-1 con rischio più elevato di eventi cardiovascolari e interruzioni precoci di gravidanza (9). La contemporanea presenza di queste 3 alterazioni genetiche predisponeva fortemente il nostro paziente a eventi cardiovascolari in generale e trombotici in particolare.

È da rilevare che, prima del manifestarsi della vasculite, non si era mai verificato alcun evento trombotico o tromboflebitico. Tale riscontro, che a noi può apparire inusuale, non è però descritto in letteratura come raro. La predisposizione all'evento trombotico può rimanere allo stato latente, per poi manifestarsi in concomitanza con altre circostanze favorenti la trombosi quali possono essere state, nel caso del nostro paziente, probabilmente la vasculite e sicuramente la terapia cortisonica.

Abbiamo purtroppo solo tardivamente effettuato i test sulla coagulazione che si sono poi conclusi con l'indagine genetica. Abbiamo trattato la vasculite con terapia immunosoppressiva ma senza alcun risultato soddisfacente per la funzione renale. Probabilmente se a una vasculite che, per definizione, è un'infiammazione con necrosi delle pareti vascolari arteriose e venose di qualsiasi calibro si associano, per mutazioni genetiche, problemi legati alla cascata della coagulazione, il danno derivante si sommerà e sarà più rapido ed esteso.

Nel nostro caso, il paziente era positivo agli anti-PR3 ANCA (c-ANCA), che rappresentano il principale marcatore sierolo- gico della Granulomatosi di Wegener, riscontrabile nel 90\% dei casi (10), ma non specifico di questa, essendo presente, anche se con minore frequenza, nella Poliangioite microscopica nel $30 \%$ dei casi.

L'eziologia di entrambe le patologie, raggruppabili tra le vasculiti ANCA-PR3, è tuttora sconosciuta, anche se è stato proposto un possibile ruolo di fattori genetici e di agenti esogeni di natura infettiva o chimica capaci di innescare anomale risposte immunitarie (11).

La sierologia del paziente deponeva per la Granulomatosi di Wegener; non veniva però segnalato in anamnesi un impegno delle vie aeree superiori. Inoltre la radiografia del torace non evidenziava i chiari noduli granulomatosi polmonari tipici della Granulomatosi di Wegener, che tuttavia spesso sono assenti. Per contro è da osservare che il coinvolgimento renale è più frequente nelle Poliangioiti. Pertanto il dubbio diagnostico persisteva.

Il trattamento messo in atto era comunque quello per le vasculiti ANCA associate, che risulta specifico e comune per entrambe le patologie $(12,13)$.

Da queste terapie e dal riscontro istologico renale di impegno perlopiù focale e segmentario, con solo un glomerulo in scleroialinosi subtotale e uno solo con rottura della capsula di Bowmann, ci aspettavamo un qualche recupero funzionale renale. Il quadro invece precipitava dopo appena 15-20 giorni dalla biopsia e il paziente restava definitivamente in dialisi.

Anche se lesioni renali severe possono essere compatibili con un outcome favorevole e lesioni modeste possono associarsi a un'evoluzione sfavorevole, ci siamo convinti che forse questa evoluzione rapida e sfavorevole è da correlare con l'habitus tromboembolico familiare presente fin dall'inizio ed evidenziato purtroppo in ritardo.

È da rilevare che le complicanze trombotiche si sono verificate quando il titolo ANCA era sensibilmente ridotto; potrebbe essere pertanto verosimile che le alterazioni genetiche, più che la vasculite stessa, abbiano favorito gli eventi trombotici.

La necessità di sottoporre a screening anche i familiari dei pazienti affetti da trombofilia ereditaria è molto controversa (14). Nella maggior parte degli studi lo si ritiene inutile.

Infatti l'identificazione di una trombofilia ereditaria in un soggetto asintomatico non potrà comportare la decisione di iniziare una terapia eparinica. È stato infatti rilevato che il rischio di eventi emorragici è decisamente superiore al rischio trombotico. Per cui il rapporto costo-beneficio, anche considerando gli alti costi dei test genetici, è sfavorevole.

Nel nostro caso specifico lo abbiamo comunque effettuato, consentendo di riscontrare in due figlie 2 delle 3 alterazioni genetiche presenti nel padre. Grazie alla terapia a base di eparina più acido folico, una figlia è riuscita a portare a termine la gravidanza in atto, mentre aveva interrotto per aborto la precedente gestazione.

\section{Conclusioni}

Nel nostro paziente il quadro "vasculitico ANCA associato" si è sviluppato su un "habitus trombofiliaco geneticamente determinato". Pertanto il solo trattamento della vasculite si è rilevato inefficace per il recupero della funzione renale. 
Se si osserva che le complicanze trombotiche si sono verificate quando il titolo ANCA era sensibilmente ridotto, è verosimile che le alterazioni genetiche, più che la vasculite stessa, abbiano favorito gli eventi trombotici.

La concomitanza di quest'altra patologia ha probabilmente inciso sfavorevolmente sulla prognosi renale ed è stato elemento confondente per il quadro clinico.

\section{Riassunto}

Descriviamo il caso clinico di un paziente giunto alla nostra osservazione per un'insufficienza renale ingravescente da sospetta vasculite. I nostri accertamenti sia laboratoristici che istologici hanno confermato la vasculite con una diagnosi clinica dubbia tra la Granulomatosi di Wegener e la Poliangioite Microscopica. Tale dubbio diagnostico non influenzava la terapia che, in base alle Linee Guida, è la stessa per le due patologie. Il paziente era trattato con Metilprednisolone, Ciclofosfamide e Plasmaferesi. Non vi è stato però alcun risultato clinico soddisfacente sulla funzionalità renale. Si è dovuta iniziare la dialisi. In questa fase si sono manifestati ripetuti episodi trombotici, che non si erano mai evidenziati prima, per cui abbiamo effettuato esami ematochimici sulla coagulazione e sulla trombosi che hanno evidenziato iperomocisteinemia. Abbiamo pertanto effettuato test genetici da cui è risultata "Trombofilia familiare" correlata a 3 alterazioni genetiche. Il trattamento a base di eparina e acido folico ha consentito di evitare il ripetersi di eventi trombotici. In conclusione, nel nostro paziente, il quadro "vasculitico ANCA associato" si è sviluppato su un "habitus trombofiliaco geneticamente determinato". Pertanto il solo trattamento della vasculite si è rilevato inefficace. La concomitanza di quest'altra patologia ha probabilmente inciso sfavorevolmente sulla prognosi renale e la sua presenza è stata un elemento confondente per il quadro clinico.

Dichiarazione di conflitto di interessi: Gli Autori dichiarano di non avere conflitto di interessi.

Contributi economici agli Autori: Gli Autori dichiarano di non aver ricevuto sponsorizzazioni economiche per la preparazione dell'articolo.

Indirizzo degli Autori:

Dr. Giovanni Salvati

U.O.C. Di Nefrologia e Dialisi

Ospedale dei Pellegrini

Via Portamedina alla Pignasecca 41

80134 Napoli

giosal1955@libero.it

\section{Bibliografia}

1. Fuiano G, Zoccali C. Linee Guida sulle indicazioni e sull'esecuzione della biopsia renale percutanea e sulla terapia delle nefropatie glomerulari. G Ital Nefrol. 2003; Suppl. 24: S3-47.

2. Sinico RA, Sabadini E. Nuove acquisizioni in tema di terapia delle vasculiti ANCA-associate.G Ital Nefrol. 2006; 2: 138-48.

3. Walsh M, Catapano F, Szpirt W. Plasma exchange for renal vasculitis and idiopathic rapidly progressive glomerulonephrits: a meta-analysis. Am J Kidney Dis. 2011; 57: 566-74.

4. Rees DC, Cox M, Clegg JB. World distribution of factor V Leiden. Lancet. 1995; 346: 1133-4.

5. Folsom AR, Cushman M, Tsai MY, et al. A prospective study of venous thromboembolism in relation to factor $\mathrm{V}$ Leiden and related factors. Blood. 2002; 99: 2720-5.

6. Davies KA, Ireland H, Athanassiou P, Loizou S, Lane D, Walport MJ. Factor V Leiden mutation and venous thrombosis. Lancet. 1995; 345: 132-3.

7. Dahlback B. Inherited thrombophilia: resistance to activated protein $\mathrm{C}$ as a pathogenic factor of venous thromboembolism. Blood. 1995; 85: 607-14.

8. Key NS, McGlennen RC. Hyperhomocyst(e)inemia and thrombophilia. Arch Pathol Lab Med. 2002; 126: 1367-75.

9. Zöller B, García de Frutos P, Dahlbäck B. A common $4 \mathrm{G}$ allele in the promoter of the plasminogen activator inhibitor-1 (PAI-1) gene as a risk factor for pulmonary embolism and arterial thrombosis in hereditary protein S deficiency. Thromb Haemostas. 1998; 79: 802-7.

10. Lamprecht P, Gross WL. Wegener's granulomatosis. Herz. 2004; 29: 47-56.

11. Groot K, Reinhold-Keller E. Wegener's granulomatosis and microscopic polyangiitis. Z Rheumatol. 2009; 68: 49-63.

12. Jayne D. Challenges in the management of microscopic polyangiitis: past, present and future. Curr Opin Rheumatol. 2008; 20: 3-9.

13. Mukhtyar C, Guillevin L, Cid MC, et al. EULAR recommendations for the management of primary small and medium vessel vasculitis. Ann Rheum Dis. 2009; 68: 310-7.

14. Baglin T. Management of thrombophilia: who to screen? Pathophysiol Haemost Thromb. 2003; 33: 401-4. 


\section{TEST DI VERIFICA}

1) Con il termine di vasculiti si intendono condizioni morbose caratterizzate istologicamente da infiammazione e necrosi delle pareti vasali che interessano:

a) Arterie e vene di qualsiasi calibro e di qualsiasi distretto, con conseguenze cliniche diverse a seconda di entità, estensione, distretto e sede dell'alterazione vasale e del calibro del vaso colpito

b) Solo arterie

c) Solo vene

d) Solo i piccoli vasi

2) Gli ANCA anti-PR3 e gli ANCA anti-MPO sono strettamente associati alla granulomatosi di Wegener, alla sindrome di Churg-Strauss, alla poliangioite microscopica e alla granulomatosi necrotizzante idiopatica rapidamente progressiva con semilune.

E possibile fare diagnosi differenziale tra Wegener e poliangioite microscopica con i soli ANCA?

a) Sì, perché gli ANCA anti-PR3 (c-ANCA) rappresentano i principali marcatori sierologici della granulomatosi di Wegener, mentre gli ANCA anti-MPO (p-ANCA) rappresentano i marcatori della poliarterite microscopica e della glomerulonefrite rapidamente progressiva

b) Sì, ma solo quando sono positivi entrambi con diversa in- tensità e associati a un aumento degli indici infiammatori

c) No, perché gli ANCA (c- e p-) sono riscontrabili in tutte le vasculiti

d) No, perché, anche se gli ANCA anti-PR3 si osservano nel 90\% delle granulomatosi di Wegener in fase attiva e gli ANCA anti-MPO nel 69-70\% delle poliangioiti microscopiche, non sono esclusivi di queste malattie

3) La trombofilia familiare è una malattia tromboembolica venosa multifattoriale, dove l'evento trombotico:

a) È il risultato di interazioni gene-gene e gene-ambiente (come le abitudini alimentari o i fattori di rischio occasionali e come un intervento chirurgico, un trauma, la gravidanza, il puerperio, l'assunzione di contraccettivi orali o le terapie estro-progestiniche)

b) Se ereditato inevitabilmente porta a morte i pazienti portatori

c) La trombosi venosa non è mai complicata da tromboembolismo

d) Sono vere la prima e la terza risposta

Le risposte corrette alle domande sono pubblicate su questo numero del Giornale di Tecniche Nefrologiche \& Dialitiche Vol. 26, no. 3, pag. 273. 